\title{
Risk factors for the development of hematological toxicity during the application of weekly paclitaxel in breast cancer
}

\author{
Alberto Alvarado-Miranda1, Germán Calderillo-Ruiz², Rafael Rodríguez-Ortiz³ , Lenny Gallardo4, \\ Karla Itzel Aguilar-Flores ${ }^{5}$ and Paula Cabrera-Galeana ${ }^{1 *}$
}

${ }^{1}$ Breast Medical Oncology Unit, National Cancer Institute; ${ }^{2}$ Internal Medicine Division, National Cancer Institute; ${ }^{3}$ Army and Air Force University; ${ }^{4}$ Clinical Research Division, National Cancer Institute,; ${ }^{5}$ Santa Monica, Angeles Hospital. Ciudad de México, Mexico

\begin{abstract}
Background: Paclitaxel increases survival in breast cancer. Weekly application is the best tolerated and most effective. One in 5 patients will have neutropenia and one in 15 patients will develop febrile neutropenia. The objective was to identify risk factors for the development of grade III-IV neutropenia. Material and methods: We retrospectively analyzed 257 patients who used weekly paclitaxel and had blood biometry before the weekly infusion for 12 weeks. We determined age, clinical stage, comorbidity, body mass index, weekly total infused dose, dose/m2 of body surface, number of medical consultations, visits to the emergency room and hospitalizations. Results: A total of 118 patients (45.9\%) received neoadjuvant paclitaxel, adjuvant $86(33.4 \%)$ and 53 first-line palliative therapy (20.6\%). The frequency of neutropenia grade III-IV was $5.9 \%$. The multivariate analysis identified as independent factors for neutropenia grade III-IV: age (over 60 years) (OR: 3.11; Cl 95\%: 1.05-9.20; $p=0.04$ ), previous chemotherapy (OR: 3.74; Cl 95\%: 1.15-12.1; $p=0.027$ ), being hypertensive (OR: $6.63 ; \mathrm{Cl} 95 \%$ : 2.18-20.15; $p=0.001$ ). Conclusions: In patients older than 60 years, exposition to previous chemotherapy and some comorbidities, particularly hypertension, are risk factors for the development of grade III-IV neutropenia. The low incidence of grade III-IV neutropenia in daily clinical practice during weekly paclitaxel suggests a reconsideration of the weekly evaluation with blood biometry in patients without risk factors.
\end{abstract}

Key words: Weekly paclitaxel. Breast cancer. Hematologic toxicity.

Correspondence:

*Paula Cabrera-Galeana

E-mail: drapaulacabrera@gmail.com
Available online: 04-07-2019 Gac Mex Oncol. 2019;18:8-12

www.gamo-smeo.com 


\section{Introduction}

Paclitaxel increases survival in breast cancer advanced and early stages. It stabilizes and polymerizes the microtubules by binding to the tubulin beta subunit, which generates cell-death during the mitosis phase ${ }^{1,2}$. Since its appearance, paclitaxel has been assessed at different doses, infusion times and administration periods. Five prospective phase III studies have shown that weekly administration is the best tolerated and effective schedule ${ }^{1,2}$. However, this schedule is considered inconvenient due to an increased use of infusion room time, outpatient services and laboratory saturation and an increase in indirect costs ${ }^{3,4}$.

The weekly adjuvant and neoadjuvant modality is administered for 12 consecutive weeks at a dose of $80 \mathrm{mg} / \mathrm{m}^{2}$ in a one-hour infusion ${ }^{5}$. Investigations have identified that one out of every five patients will experience neutropenia and one in 15 will develop febrile neutropenia².

Despite the widespread use of this regimen in our country, few studies have assessed hematological toxicity outside the setting of a research protocol, whose safety data are difficult to transfer to the unselected population of daily clinical practice ${ }^{3}$.

There is no clear recommendation for the follow-up of weekly paclitaxel hematological toxicity. Our objective was to identify risk factors for the development of grade III-IV neutropenia and to characterize a neutropenia behavioral pattern during the twelve weeks of administration that allows recommending an assessment interval for patients receiving this type of treatment.

\section{Material and methods}

A retrospective sample of patients with breast cancer confirmed diagnosis between 2011 and 2015 was selected. Those who received weekly paclitaxel for at least 12 weeks and who had a blood count available for each administration week were included. Blood count had to be performed within a period no longer than 48 hours prior to infusion. The weekly paclitaxel indication was allowed in the neoadjuvant and adjuvant setting, regardless of the anthracycline sequence. In advanced disease, only cases on first line-treatment were included. Patients with HER2 overexpression received trastuzumab. Demographic, clinical and laboratory data were obtained from the National Institute of Cancerol electronic records. Age, clinical stage, HER2 status, comorbidity, body mass index (BMI), total weekly-infused dose and dose per body surface area square meter were identified. The number to outpatient visits, visits to the emergency room and patient hospitalizations were also recorded during the 12 weeks of paclitaxel treatment.

Hematological adverse events were classified according to the Common Toxicity Criteria for Adverse Events (CTCAE) guidelines, version 4.03. The highest CTCAE grade was obtained for each type of hematological adverse event and for each patient. The proportions of patients with any hematological event were compared using the chi-square test. The patterns of the most common hematological adverse events were assessed over time. A descriptive analysis of demographic variables was also carried out. We measured the relationship of neutropenia with risk factors for its development using univariate and multivariate analyses.

\section{Results}

Data of a total of 257 patients, in whom mean age was 52 years (21 to 87 years) were analyzed. There were 44 cases (17.1\%) of early stage disease (I-IIA), 161 patients $(62.6 \%)$ had locally advanced disease (IIB-IIIC), and distant disease was observed in 53 patients $(20.6 \%)$. HER2 overexpression was found in $22.5 \%$ (58) of patients. Weekly paclitaxel was used as neoadjuvant therapy in 118 patients (45.9\%), as adjuvant therapy in $86(33.4 \%)$ and as first-line palliative therapy in 53 patients $(20.6 \%)$. BMI, comorbidity, number of patient visits and dose intensity are reported in table 1. Most common hematological toxicities per patient during the twelve assessment weeks are presented in table 2, with the absence of febrile neutropenia cases and a rate lower than $1 \%$ for grade IV neutropenia, leukopenia and thrombocytopenia standing out. The frequency of grade III and IV neutropenia was $5.9 \%$. Dose adjustment was required by $20 \%$ of patients due to non-hematological toxicity.

Seventy patients attended immediate care services at least once and 21 patients were hospitalized during the paclitaxel administration 12-week period. None of these visits and hospital admissions were associated with hematologic toxicity.

The univariate analysis is presented in table 3. BMI higher than 25, total dose higher than $140 \mathrm{mg}$ per week, the administration clinical setting, and the number of patient visits, hospitalizations for other toxicities and visits to the emergency room were not correlated with the risk for developing grade III-IV neutropenia.

In the multivariate logistic regression analysis, the following independent variables for the development of grade III and IV neutropenia were identified: age (older 
Table 1. Demographic and oncological treatment characteristics of breast cancer patients included in the study who received weekly paclitaxel

\begin{tabular}{|c|c|c|c|}
\hline Age & 52-year mean (21-87) & $\mathbf{n}$ & $(\%)$ \\
\hline \multirow[t]{2}{*}{$\mathrm{BMI}$} & $<25$ & 74 & (28.7) \\
\hline & $>25$ & 183 & (71.2) \\
\hline \multirow[t]{5}{*}{ Comorbidities } & DM2 & 20 & $(7.7)$ \\
\hline & $\mathrm{HBP}$ & 49 & (19) \\
\hline & HBP/DM2 & 10 & $(3.9)$ \\
\hline & Other comorbidities & 20 & $(7.7)$ \\
\hline & No comorbidities & 158 & (61.8) \\
\hline \multirow[t]{3}{*}{ Clinical stage } & Early (I, IIA) & 44 & (17.1) \\
\hline & $\begin{array}{l}\text { Locally } \\
\text { advanced (IIB-IIIC) }\end{array}$ & 161 & (62.6) \\
\hline & Metastatic (IV) & 53 & (20.6) \\
\hline \multirow[t]{2}{*}{ Treatment modality } & Neoadjuvant/adjuvant & 204 & (79.4) \\
\hline & First-line palliative & 53 & (20.6) \\
\hline \multirow[t]{2}{*}{ Regimen } & Paclitaxel & 199 & (77.5) \\
\hline & Paclitaxel + trastuzumab & 58 & (22.5) \\
\hline \multirow[t]{3}{*}{ Dose intensity } & $<75 \mathrm{mg} / \mathrm{m}^{2}$ & 27 & (10.5) \\
\hline & $75-79.9 \mathrm{mg} / \mathrm{m}^{2}$ & 177 & (68.9) \\
\hline & $\geq 80 \mathrm{mg} / \mathrm{m}^{2}$ & 53 & (20.6) \\
\hline \multirow[t]{3}{*}{ No. of patient visits } & $<4$ & 46 & (18.6) \\
\hline & $5-7$ & 167 & (64.9) \\
\hline & $>8$ & 44 & (17.1) \\
\hline
\end{tabular}

BMI: body mass index.

than 60 years, OR: $3.11,95 \% \mathrm{Cl}: 1.05-9.20, \mathrm{p}=0.04)$, having received previous chemotherapy (OR: $3.74,95 \%$ $\mathrm{Cl}$ : 1.15-12.1, $p=0.027$ ) and being hypertensive (OR: 6.63, 95\% Cl: 2.18-20.15, $p=0.001$ ).

\section{Discussion}

The variability of grade III and IV neutropenia rates in published prospective controlled studies in the three clinical settings (neoadjuvant, adjuvant and metastatic) has generated different clinical practices in hematological toxicity assessment ${ }^{5-10}$.

The frequency at which blood count should be performed prior to paclitaxel off-protocol weekly infusion has not been described. In the study by Sparano et al. ${ }^{5}$, which included 1,231 patients who received weekly adjuvant paclitaxel, blood count was obtained every week prior to the infusion (and this behavior has therefore become generalized) and the percentage of grade III-IV neutropenia was $2 \%$. However, Seidman et al. ${ }^{11}$ performed a blood count every three weeks in 572 patients with advanced disease, and the frequency of grade III and grade IV neutropenia was $5 \%$ and $3 \%$, respectively, which is why some clinicians also use this practice. These results are in contrast with those reported by Ando et al. ${ }^{12}$, who assessed 181 patients who received weekly neoadjuvant paclitaxel followed by anthracyclines, and identified $8.8 \%$ of grade III and $1.1 \%$ of grade IV neutropenia, while in Miller et al. ${ }^{6}$ study in 346 patients with metastatic disease who received weekly paclitaxel as first-line of treatment, grade III neutropenia was $0.3 \%$ and no patient experienced grade IV neutropenia. It is difficult to determine an exact risk fot hematological toxicity.

Our study reports a frequency of grade III neutropenia of $5.1 \%$ and grade IV of $0.4 \%$ in unselected patients from the usual clinical practice. These percentages confirm the hematological safety of paclitaxel weekly administration in our daily practice. The result is similar to the $5.7 \%$ rate of grade III-IV neutropenia reported in a Brazilian population exposed to off-clinical protocol weekly paclitaxel ${ }^{3}$.

The percentage of patients who have dose reductions when using this regimen for metastatic disease has been reported to be $27 \%$ and $29 \%$ in patients on adjuvant treatment ${ }^{5,12,13}$, with neurotoxicity being the main dose-limiting toxicity ${ }^{2}$. In our data, dose reduction was identified in $20 \%$ of patients due to non-hematological toxicities; this value may be influenced by population characteristics, including the fact of being on adjuvant, neoadjuvant and first-line palliative therapy. On the other hand, $89.5 \%$ of the population maintained a dose intensity above $75 \mathrm{mg} / \mathrm{m}^{2} /$ week despite the fact that 1 out of each 5 patients had advanced disease, which comparable with the mean dose intensity of $77 \mathrm{mg} / \mathrm{m}^{2} /$ week reported outside the setting of clinical trials for early disease ${ }^{3}$. According to a previous publication of our group, this $10.5 \%$ of patients with a dose intensity lower than $75 \mathrm{mg} / \mathrm{m}^{2} /$ week does not negatively affect our population survival ${ }^{14}$.

We did not find previous studies identifying risk factors for the development of hematological toxicity during the administration of weekly paclitaxel. In the multivariate analysis, our results clearly show three independent factors for the development of grade III and IV neutropenia: age older 60 years, presence of hypertension and previous exposure of the patient to 
Table 2. Hematological toxicity per patient during the 12 weeks of paclitaxel weekly administration in patients with breast cancer assessed in the study

\begin{tabular}{|l|c|c|c|c|c|}
\hline Toxicity & Grade I n (\%) & Grade II n (\%) & Grade III n (\%) & Grade IV n (\%) & No toxicity n (\%) \\
\hline Neutropenia & $47(18.3)$ & $29(11.3)$ & $13(5.1)$ & $1(0.4)$ & $167(65)$ \\
\hline Leukopenia & $97(37.7)$ & $42(16.3)$ & $11(4.3)$ & $2(0.8)$ & $105(40.9)$ \\
\hline Anemia & $85(33.1)$ & $18(7.0)$ & $1(0.4)$ & $0(0)$ & $153(59.5)$ \\
\hline Thrombocytopenia & $27(10.5)$ & $6(2.3)$ & $0(0)$ & $0(0)$ & $224(87.2)$ \\
\hline Lymphopenia & $93(36.2)$ & $41(16)$ & $10(3.9)$ & $4(1.6)$ & $109(42.4)$
\end{tabular}

Table 3. Univariate analysis to assess risk factors for the development of hematological toxicity during paclitaxel weekly administration

\begin{tabular}{|c|c|c|c|}
\hline Variable & OR & $\begin{array}{l}95 \% \text { confidence } \\
\text { interval }\end{array}$ & $\mathbf{p}$ \\
\hline Age $<60$ years & 0.256 & $0.103-0.633$ & 0.04 \\
\hline Age $>60$ years & 4.453 & $1.632-12.149$ & 0.04 \\
\hline $\mathrm{BMI}>25$ & 0.818 & $0.278-2.402$ & NS \\
\hline $\begin{array}{l}\text { Total dose higher than } 140 \\
\text { mg }\end{array}$ & 1.677 & $0.188-2.440$ & NS \\
\hline $\begin{array}{l}\text { Patients with }>8 \text { outpatient } \\
\text { visits }\end{array}$ & 0.473 & $0.176-1.273$ & NS \\
\hline Prior chemotherapy & 442 & $1.144-8.810$ & 0.01 \\
\hline $\begin{array}{l}\text { Neoadjuvant or adjuvant } \\
\text { treatment }\end{array}$ & 1.11 & $0.810-1.520$ & NS \\
\hline $1^{\text {st }}$ line palliative treatment & 0.743 & $0.340-1.610$ & NS \\
\hline $\begin{array}{l}\text { Presence of } \\
\text { comorbidities (DM, obesity, } \\
\text { HBP) }\end{array}$ & 6.419 & 2.027-20.322 & 0.04 \\
\hline High blood pressure & 5.805 & 2.099-16.055 & 0.01 \\
\hline & & & NS \\
\hline Hospitalization & 5.805 & $0.086-5.407$ & NS \\
\hline
\end{tabular}

some type of chemotherapy. Similarly to us, Lichtman et al. refer that patients older than 65 years have a higher risk for developing hematological toxicity associated with weekly paclitaxel, and this risk was also increased if patients had previously received chemotherapy ${ }^{15}$. On the other hand, Barcenas et al. determined that older patients who used doxorubicin/ cyclophosphamide followed by weekly paclitaxel during adjuvant therapy had an OR of 1.80 (1.18-2.76) for chemotherapy-associated hospital admission. They also described that a Charlson index higher than 2 points conferred an OR of 2.70 (2.03-3.59). Although these hospitalizations do not only include hematological toxicity, they reinforce our results by establishing age, comorbidity and prior chemotherapy as factors associated with chemotherapy-related hematological toxicity $^{16}$.

The 6.63 OR (95\% Cl: 2.18-20.15; $p=0.001)$ for hypertension stands out in our study. Although it has not been previously reported, this phenomenon can be explained by the fact that $38.2 \%$ of analyzed patients had some type of comorbidity and $59.5 \%$ of them they had hypertension, alone or associated with other comorbidity (DM2/obesity).

The retrospective nature of the study confers limitations to it. In prospective protocols, the use of colony-stimulating factor is allowed; we do not have exact data on the use of colony stimulating factor used in our population, which might explain why we failed to identify cases with febrile neutropenia, despite the fact that published rates range from $0.1 \%$ to $1 \%$ when weekly paclitaxel is used in the adjuvant setting ${ }^{5,8}$. The heterogeneity in the management of hematological adverse events by clinicians was not documented and, therefore, like other retrospective studies, we have a registration and selection bias, since the decision to indicate and administer the treatment was not controlled ${ }^{9}$.

One of our objectives was to recognize a behavioral pattern of neutropenia, but its low incidence hindered meeting this purpose, and it is therefore not possible to make recommendations on the ideal blood count timing for hematological toxicity general assessment.

However, given that most oncology services in our country are saturated, we believe that identifying a high-risk group might help optimize human and laboratory resources since, even if weekly paclitaxel is used without prior exposure to chemotherapy, the rates of grade III-IV neutropenia drop to 3.0 and $4.2 \% 17,18$. 
Performing a blood count every three weeks seems to be a good practice in the absence of risk factors, since Seidman's study, which included patients with advanced disease, recorded a rate of grade III-IV neutropenia of 8 and $65 \%$ in patients who had previously received chemotherapy ${ }^{11}$.

The findings of our study might contribute to implement prioritization strategies in order to free spaces in the oncology department, to reduce the taking of laboratory samples and to decrease direct and indirect costs, since paclitaxel weekly administration increases the costs of care ${ }^{4}$.

\section{Conclusions}

The risk factors for the development of grade III-IV neutropenia are age older than 60 years, prior exposure to chemotherapy, comorbidity and hypertension. This group of patients benefits from close hematological surveillance. The probability of experiencing hematological adverse effects secondary to paclitaxel weekly administration in daily clinical practice is low. Weekly evaluation with blood count could be reconsidered. An evaluation with blood count every three weeks is recommended in the absence of said factors.

\section{Acknowledgements}

The authors express their gratitude to doctors Rebeca Ramírez, Jorge Alegría, Josana Rodríguez, Claudia Arce, Fernando Lara and Manuel Magallanes.

\section{References}

1. von Minckwitz G, Martin M, Wilson G, Alba E, Schmidt M, Biganzoli L, et al. Optimizing taxane use in $M B C$ in the emerging era of targeted chemotherapy. Crit Rev Oncol Hematol. 2013;85(3):315-31.

2. Carbognin L, Sperduti I, Nortilli R, Brunelli M, Vicentini C, Pellini F, et al. Balancing activity and tolerability of neoadjuvant paclitaxel- and docetaxel-based chemotherapy for HER2-positive early stage breast cancer: Sensitivity analysis of randomized trials. Cancer Treat Rev. 2015;41(3): 262-70.
3. Santana IA, Oliveira JA, da Silva Lima JM, Testa L, Piato JR, Hoff PM, et al. Feasibility of two schedules of weekly paclitaxel in HER2-negative early breast cancer in a Brazilian community setting. Breast Cancer 2016;23(2):261-5

4. Frías C, Cortés J, Seguí MÁ, Oyagüez I, Casado MÁ. Cost-effectiveness analyses of docetaxel versus paclitaxel once weekly in patients with metastatic breast cancer in progression following anthracycline chemotherapy, in Spain. Clin Transl Oncol. 2010;12:692-700.

5. Sparano JA, Wang M, Martino S, Jones V, Perez EA, Saphner T, et al. Weekly paclitaxel in the adjuvant treatment of breast cancer. $\mathrm{N}$ Engl J Med. 2008;358(16):1663-71.

6. Miller K, Wang M, Gralow J, Dickler M, Cobleigh M, Perez EA, et al. Paclitaxel plus bevacizumab versus paclitaxel alone for metastatic breast cancer. N Engl J Med. 2007;357(26):2666-76.

7. Qi WX, Shen Z, Lin F, Sun YJ, Min DL, Tang LN, et al. Paclitaxel-based versus docetaxel-based regimens in metastatic breast cancer: a systematic review and meta-analysis of randomized controlled trials. Curr Med Res Opin. 2013;29(2):117-25.

8. Budd GT, Barlow WE, Moore HCF, Hobday TJ, Stewart JA, Isaacs C, et al. SWOG S0221: A phase III trial comparing chemotherapy schedules in high-risk early-stage breast cancer. J Clin Oncol. 2015;33(1): 58-64.

9. Alsharedi M, Gress T, Dotson J, Elmsherghi N, Tirona MT. Comparison of toxicity profile and tolerability between two standard of care paclitaxel-based adjuvant chemotherapy regimens in breast cancer. Med Oncol. 2016:33(3):1-5.

10. Gonzalez-Angulo AM, Akcakanat A, Liu S, Green MC, Murray JL, Chen $\mathrm{H}$, et al. Open-label randomized clinical trial of standard neoadjuvant chemotherapy with paclitaxel followed by FEC versus the combination of paclitaxel and everolimus followed by FEC in women with triple receptor-negative breast cancer. Ann Oncol. 2014;25(6): 1122-7.

11. Seidman AD, Berry D, Cirrincione $C$, et al. Randomized phase III trial of weekly compared with every-3-weeks paclitaxel for metastatic breast cancer, with trastuzumab for all HER-2 overexpressors and random assignment to trastuzumab or not in HER-2 nonoverexpressors: Final results of cancer and leu. J Clin Oncol. 2008;26(10):1642-9.

12. Ando M, Yamauchi H, Aogi K, et al. Randomized phase II study of weekly paclitaxel with and without carboplatin followed by cyclophosphamide/ epirubicin/5-fluorouracil as neoadjuvant chemotherapy for stage II/IIA breast cancer without HER2 overexpression. Breast Cancer Res Treat. 2014;145(2):401-9.

13. John M, Hinke A, Stauch M, et al. Weekly paclitaxel plus trastuzumab in metastatic breast cancer pretreated with anthracyclines-a phase II multipractice study. BMC Cancer. 2012;12(1):165.

14. Reynoso-Noverón N, Villareal-Garza C, Soto-Perez-de-Celis E, Arce-Salinas C, Matus-Santos J, Ramírez-Ugalde MT, et al. Clinical and epidemiological profile of breast cancer in Mexico, results of the Seguro Popular. J Glob Oncol. 2017;3(6):757-64.

15. Lichtman SM, Hurria A, Cirrincione CT, et al. Paclitaxel efficacy and toxicity in older women with metastatic breast cancer: Combined analysis of calgb 9342 and 9840. Ann Oncol. 2012;23(3):632-8.

16. Barcenas $\mathrm{CH}$, Niu J, Zhang N, et al. Risk of hospitalization according to chemotherapy regimen in early-stage breast cancer. J Clin Oncol. 2014; 32(19):2010-7.

17. Tolaney SM, Barry WT, Dang CT, et al. Adjuvant paclitaxel and trastuzumab for node-negative, HER2-positive breast cancer. N Engl J Med. 2015;372(2):134-41.

18. Shulman LN, Berry DA, Cirrincione CT, et al. Comparison of doxorubicin and cyclophosphamide versus single-agent paclitaxel as adjuvant therapy for breast cancer in women with 0 to 3 positive axillary nodes: CALGB 40101 (alliance). J Clin Oncol. 2014;32(22):2311-7. 